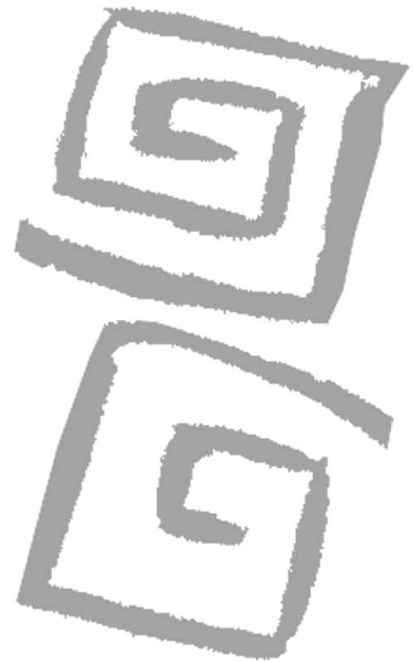

\title{
Sustancias consideradas adictivas: prohibición, reducción de daños y reducción de riesgos
}

\author{
Substances considered addictive: prohibition, \\ harm reduction and risk reduction
}

Menéndez, Eduardo L. ${ }^{1}$

${ }^{1}$ Doctor en Ciencias Antropológicas. Profesor e investigador, Centro de Investigaciones y Estudios Superiores en Antropología Social (CIESAS), México. Coordinador del Seminario Permanente de Antropología Médica (SPAM).

emenendez1@yahoo.com.mx
RESUMEN América Latina es actualmente la región con mayor tasa de homicidios a nivel mundial, y gran parte de los asesinatos están vinculados al denominado crimen organizado, especialmente al narcotráfico. El narcotráfico es producto de la ilegalización de ciertas sustancias, la cual -por lo menos en la actualidad- está basada y legitimada en criterios biomédicos que convierten la producción, comercialización y frecuentemente el consumo de ciertas sustancias consideradas adictivas en "delitos contra la salud". En este texto se analizan en forma sucinta las dos políticas formuladas y aplicadas hasta ahora en términos prohibicionistas y de reducción de daños, considerando el fracaso del prohibicionismo, así como las limitaciones de las propuestas de reducción de daños. Respecto del prohibicionismo se señalan sus múltiples y constantes incongruencias y contradicciones, lo cual implica que tomemos con cautela los reiterados señalamientos sobre su "fracaso". El texto propone la aplicación de una política de reducción de riesgos que incluya no solo los comportamientos de sujetos y grupos, sino también la dimensión estructural tanto en términos económico-políticos como culturales.

PALABRAS CLAVE Sustancias Consideradas Adictivas; Políticas Prohibicionistas; Reducción del Daño; Reducción del Riesgo; Homicidio.

ABSTRACT Latin America is currently the region with the highest rate of homicides worldwide, and a large part of the killings are linked to so-called organized crime, especially drug trafficking. The trafficking of drugs is a consequence of the illegality of certain substances which - at least presently - is based in and legitimated by biomedical criteria that turns the production, commercialization and often the consumption of certain substances considered addictive into "offenses against health." This text briefly analyzes the two policies formulated and implemented thus far in terms of prohibition and harm reduction, considering the failure of prohibitionism as well as the limitations of harm reduction proposals. The constant and multiple inconsistencies and contradictions of prohibitionism are noted, indicating the necessity of regarding cautiously repeated comments about its "failure." The text proposes the implementation of a policy of risk reduction that includes not only the behavior of individuals and groups, but also the structural dimension, both in economic-political and cultural terms.

KEY WORDS Substances Considered Addictive; Prohibitionist Policies; Harm Reduction; Risk Reduction; Homicide. 


\section{INTRODUCCIÓN}

Uno de los problemas sociales más graves, que se está incrementando en casi toda América Latina, lo constituye la expansión del denominado crimen organizado y, especialmente, del narcotráfico. El crimen organizado opera como una empresa capitalista, cumpliendo no solo los deseos, necesidades y/o demandas de los diferentes tipos de usuarios, sino gran parte de las reglas de una sociedad consumista. De tal manera que provee drogas consideradas adictivas a los sujetos que las demandan, prostitución femenina y masculina para satisfacer los deseos sexuales de los compradores y toda una variedad de órganos para trasplantes que son realizados por personal médico. Además, el crimen organizado crea constantemente puestos de trabajo -incluido el trabajo de sicario- en varias sociedades caracterizadas por las altas tasas de desocupación y por los bajos salarios que recibe la mayoría de la población.

Si bien las acciones del crimen organizado tienen consecuencias de diferente tipo, por el momento me interesa señalar solo una, y es que especialmente el narcotráfico no solo ha generado y/o ha intervenido en toda una variedad de violencias, sino que se ha constituido en una de las principales causas de homicidio, hasta convertir a ciertos países americanos en aquellos que tienen las más altas tasas de homicidio a nivel mundial. Más aun: ha conducido -junto con otros procesos- a que determinadas regiones de América sean las más homicidas del planeta (a).

Debemos recordar que actualmente el narcotráfico se sustenta en un hecho básico: la conversión en ilegales, y por lo tanto la prohibición, de gran parte de las sustancias consideradas adictivas, siendo esta prohibición la que impulsa el desarrollo creciente del narcotráfico y posibilita -y subrayo lo de posibilita- el incremento de los homicidios (b).

Si bien la ilegalidad es condición necesaria para que haya narcotráfico, ello no supone que toda producción y comercialización ilegal de las drogas consideradas adictivas, implique las altas tasas de homicidios que observamos actualmente, especialmente en países como Brasil, Colombia, El Salvador, Guatemala o México. Pero este aspecto -aun siendo relevante- no lo desarrollaré, porque no forma parte de los objetivos centrales de este trabajo.

Ahora bien, ¿en qué se basa la ilegalidad de las sustancias consideradas adictivas? Se basa en consideraciones morales, políticas, racistas, pero -por lo menos en la actualidad- se basa también en criterios biomédicos. Es la biomedicina la que establece profesionalmente que estas sustancias tienen consecuencias nocivas para la salud, es decir, que pueden generar padecimientos tanto a nivel individual como colectivo. Más aun, la biomedicina considera que la adicción es en sí un padecimiento, fundamentando "científicamente" la prohibición de estas drogas y reclamando, a través del sector salud, el uso exclusivo de las mismas por razones terapéuticas, y posibilitando que sus usos ilegales se conviertan en "delitos contra la salud" (c).

No obstante, han sido procesos económicos, políticos o sociales los que han impulsado reiteradamente las políticas prohibicionistas $(3,4)$, tratando de articularse con criterios biomédicos, pero subordinándolos. Suelen ser criterios biomédicos los que fundamentan las acciones contra las drogas, pero en las prácticas se imponen acciones penales y policiales. Al menos en las últimas tres décadas, el aparato médico-sanitario a nivel de cada país no constituye la institución que establece y, aún menos, Ileva a cabo las políticas respecto de las "drogas", sino que el eje de las políticas está en los organismos políticos de gobierno y especialmente en los de seguridad. Y lo mismo ocurre a nivel internacional, no son la Organización Mundial de la Salud (OMS) ni la Organización Panamericana de la Salud (OPS) -en el caso de América Latina- los organismos encargados de establecer las políticas que realmente se aplican respecto de los usos y consumos de drogas, pese a ser considerados como graves problemas de salud, sino que son organismos de las Naciones Unidas como la Comisión de Estupefacientes, la Junta Internacional de Fiscalización de Estupefacientes y la Oficina de las Naciones Unidas contra la Droga y el Delito, las que realmente proponen y deciden dichas políticas (d).

Lo señalado se verifica detectando quiénes son los actores sociales que planifican y Ilevan a cabo la "lucha contra las drogas", así como quiénes son los que reciben y utilizan la mayor 
cantidad de recursos de casi todo tipo, especialmente los recursos financieros que, como sabemos, son los organismos de seguridad.

Los aparatos médico-sanitarios, hasta fechas relativamente recientes, ni siquiera se hacían realmente cargo de los aspectos adictivos de estas drogas, sino que lo que atendían eran algunas de sus consecuencias $y$, sobre todo, los aspectos físicos de las mismas. No veían las adicciones como un problema médico, lo cual -en el caso del "alcoholismo"- puede observarse con claridad no tanto a nivel del discurso sino de las prácticas médicas específicas (2).

\section{POLÍTICAS Y DROGAS: PRINCIPALES CARACTERÍSTICAS Y CRÍTICAS}

En términos simplificados existirían dos propuestas de acción global respecto de las consideradas sustancias adictivas: las prohibicionistas y las de reducción de daños. Las dominantes, desde fines del siglo XIX hasta la actualidad, han sido las prohibicionistas, que son las que realmente fueron aplicadas a través de las políticas específicas de cada Estado-nación. La denominada reducción de daños se desarrolló desde mediados de la década de 1980 a través de actividades puntuales en Alemania, Australia, España, Holanda, Reino Unido, Suiza, y solo se aplica más integralmente en Portugal, donde se ha legalizado el consumo de estas sustancias. Pero subrayando que, en la última década, se expandió notoriamente la aplicación de estas actividades:

\footnotetext{
Actualmente, unos ochenta países implementan programas de intercambio de jeringas, y alrededor de un millón de personas reciben tratamientos de sustitución de heroína con metadona o bupremorfina [...] En 2003, la Unión Europea (UE) adoptó el enfoque de reducción de daños como posición común, incluyéndolo en la Estrategia de Drogas de la UE para el período 2005-2012. (5 p.335)
}

No obstante, como veremos luego, en la mayoría de los países las actividades de reducción de daños refieren a ciertos aspectos puntuales centrados en los individuos.
Como señalé, las políticas prohibicionistas son las que han dominado las estrategias mundiales respecto de las drogas consideradas adictivas, aunque reconociendo que ha habido períodos de mayor y menor intensidad en la aplicación de las medidas prohibitivas, y que existen notorias diferencias entre los países en la aplicación de dichas medidas. A partir de la década de 1970, EE.UU. generó e impulsó la denominada "guerra contra las drogas" aplicando medidas prohibitivas que trataron de imponer a nivel mundial a través de sanciones políticas y económicas. Sin embargo, dicha "guerra" está siendo perdida en todos los países donde ha sido aplicada. Justamente, el desarrollo de las actividades de "reducción de daños" constituye una de las expresiones más notorias de este fracaso prohibicionista.

A continuación presentaré las principales características y críticas de estas dos políticas en términos generales y esquemáticos, dado que tanto dentro del prohibicionismo como de la reducción de daños existen diferencias específicas y, en algunos casos, sustantivas; incluso al interior de un solo país, como es el caso de EE.UU. donde pese al prohibicionismo federal, varios estados han adoptado actividades de reducción de daños.

Las principales características de las políticas prohibicionistas son las siguientes:

a) Desde por lo menos la década de 1960, las acciones prohibitivas respecto de las drogas consideradas adictivas refirieron a criterios de adicción que, durante la década de 1970, se convertirían en criterios de "dependencia" (e).

b) Explícita o implícitamente consideran al adicto como un sujeto "dependiente" de una sustancia que determina su comportamiento $y$ que, por lo tanto, necesita ser controlado.

c) Gran parte de estas sustancias no solo son consideradas ilegales, sino que son prohibidas, por lo cual la producción, venta y frecuentemente el consumo constituyen un delito.

d) El objetivo central de estas políticas es reducir el consumo hasta eliminarlo; es decir, contribuir a producir sujetos abstemios. En México, el mensaje dominante del sector salud, de las instituciones encargadas del tratamiento de los adictos, de la Secretaría de Educación Pública, es la abstinencia. 
e) La legislación sobre estas drogas implica castigos, incluido encarcelamiento $y$, en algunos países, el ajusticiamiento.

f) Estas políticas generan criminalización, persecución, estigmatización de las drogas consideradas adictivas, y de los diferentes actores implicados en la producción, comercialización y consumo de dichas sustancias, de tal manera que se incrementan los delitos relacionados con las drogas y el encarcelamiento de los delincuentes. Y así encontramos que el $25 \%$ de las nueve millones de personas encarceladas en el mundo, lo son por ese tipo de delito (7). Más aún, un trabajo que analiza la situación para Latinoamérica concluye que "es asombroso el alto número de personas en prisión por posesión simple de drogas, incluyendo la marihuana, hasta en países donde el porte de pequeñas cantidades de drogas para consumo personal no es

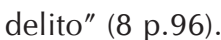

g) Las políticas prohibicionistas afectan sobre todo a los sectores sociales más pobres tanto en términos de usuarios como de operadores del narcomenudeo. Son los sectores más criminalizados, más encarcelados y más perseguidos. Son los sectores -principalmente varones adolescentes y adultos jóvenes- donde observamos el mayor número de asesinados, pero también de homicidas relacionados con el narcotráfico. Más aún, son los sectores en los cuales se observan las más graves consecuencias en términos de salud, al mismo tiempo que son los que tienen el menor acceso a los servicios médicos y psicoterapéuticos.

h) El incremento de las consecuencias en términos de mortalidad y morbilidad afecta no solo a los actores involucrados en la producción, comercialización, consumo, y en la represión, sino que genera cada vez más "daños colaterales". Las políticas punitivas favorecieron en ciertos contextos la expansión del VIH-sida, pues la prohibición convierte al consumidor en un delincuente que no demanda atención médica. Además condujo, especialmente a los sectores más pobres y excluidos, al uso de sustancias adictivas no solo de baja calidad sino con mayor efecto de deterioro, como ocurre con el denominado "paco" (pasta base de cocaína) en Argentina o con los pegamentos en México. Es decir que este tipo de políticas orienta a los sujetos y grupos al desarrollo de comportamientos cada vez más peligrosos para su salud, y a demandar muy poco o directamente no demandar atención terapéutica por varias razones, incluida la criminalización del consumo. Es importante subrayar que estudios recientes indican que -por lo menos en países latinoamericanos- hay muchas más muertes debido a la aplicación de las políticas punitivas, que por el efecto del consumo de cocaína, heroína y de otras sustancias consideradas adictivas.

i) Casi todos los países con mayor o menor rigor adhieren al prohibicionismo, más allá de su orientación político-ideológica, de sus características económico-políticas, de su mayor o menor desarrollo democrático, de sus formas religiosas dominantes. Lo cual no significa ignorar que estos aspectos pueden reducir o agudizar la aplicación de prohibiciones y castigos, y la intensidad de los mismos. Pero lo que me interesa subrayar es el dominio de estas políticas en prácticamente todos los países, lo que significa que adhieren a los principios básicos del prohibicionismo, más allá de que se impulsen las actividades de reducción de daños.

j) La aplicación de estas políticas ha posibilitado grandes ganancias en términos económicos tanto a nivel ilegal como legal, especialmente a través del Ilamado "lavado de dinero", así como favorece e impulsa los procesos de impunidad y corrupción en todos los niveles socioeconómicos. La corrupción y la impunidad constituyen procesos previos al desarroIlo actual del crimen organizado, pero que son implementados e incrementados por el desarrollo actual del narcotráfico.

k) Ha posibilitado y legitimado además el desarroIlo de aparatos jurídico-policiales y, en algunos casos, también militares, que actúan contra el denominado crimen organizado, incluidos los "drogodependientes". Estos aparatos pueden operar como fuerza de control social y político tanto a nivel de cada país, como en términos de política exterior. El notable desarrollo de los aparatos de persecución y control, especialmente en ciertos países latinoamericanos, contrasta con ciertos datos epidemiológicos, ya que según un reciente informe 
de la Organización de las Naciones Unidas (ONU), existirían a nivel mundial 208 millones de consumidores de drogas ilícitas, es decir un $4,8 \%$ de la población mundial consumiría este tipo de drogas (9). Pero además solo el 0,1\% de la población mundial consumiría drogas consideradas "duras" como la cocaína o los derivados de la cocaína (10). Por lo cual necesitamos reflexionar sobre cuál es el papel real que cumple no solo el prohibicionismo, sino especialmente la guerra contra las drogas, sin por ello negar que determinados consumos pueden generar directa o indirectamente graves problemas sociales y de salud.

I) A su vez, las políticas prohibicionistas favorecen el desarrollo de mecanismos de autocontrol social a través del miedo y de la inseguridad, convirtiéndose la seguridad en una de las principales demandas de la población de países como Colombia, Guatemala o México.

m) Todos los indicadores subrayan el fracaso de las políticas prohibicionistas en muy diversos aspectos de la realidad, ya que no solo no se redujo la producción ni el consumo, sino que incluso aumentó, sobre todo en países en los cuales el consumo de algunas de estas sustancias adictivas era históricamente bajo o muy bajo. Pero además, fracasó la policiación y militarización de la "guerra" contra las drogas, dado que no solo no redujo el consumo, sino que tampoco redujo las actividades ni el poder del crimen organizado, analizados sobre todo a nivel global. Si bien en EE.UU. se habría reducido en los últimos diez años el consumo de ciertas sustancias como cocaína y heroína, sin embargo se ha incrementado notablemente el consumo de drogas consideradas adictivas pero recetadas médicamente, lo cual indica que en términos reales el consumo de drogas siguió incrementándose.

Varios autores han planteado no reducir el análisis -y menos las acciones- de las políticas aplicadas a las drogas consideradas adictivas a la oposición prohibición/legalización, con lo que estamos en parte de acuerdo, pero considerando que esta propuesta debe ser contextualizada, es decir, debe ser referida a las características que adquiere el prohibicionismo en situaciones concretas, como es el caso de varios países latinoamericanos en los que implicaría impulsar la legalización como un objetivo prioritario, dadas las consecuencias de la criminalización de las sustancias consideradas adictivas. Más aun, sin olvidar los objetivos pragmáticos de la reducción de daños, debemos plantearnos la legalización como un objetivo ideológico, o si se prefiere imaginario, que opere en las transacciones que se desarrollan entre los diferentes actores sociales implicados en los diferentes usos de las sustancias adictivas legales e ilegales.

Las críticas al prohibicionismo podemos rastrearlas desde el momento en que se aplicaron las primeras políticas prohibicionistas a fines del siglo XIX y principios del siglo XX $\mathrm{Y}$, sobre todo, a partir de las políticas prohibicionistas respecto de las bebidas alcohólicas, que fueron aplicadas durante la década de 1920 en toda una serie de países que, como EE.UU., incluían la prohibición de la producción y comercialización de todo tipo de bebidas alcohólicas. Señalo en particular esta etapa, pues se le realizaron críticas muy similares a las que se hicieron en las últimas décadas respecto de las políticas prohibicionistas actuales, que subrayaron no solo el incremento del crimen organizado y de la mortalidad por homicidios sino, sobre todo, el fracaso de esta política dado que no consiguió su objetivo central, es decir eliminar el consumo. Lo cual condujo a derogar la prohibición de producción y venta de bebidas alcohólicas en casi todos los países "occidentales".

A mediados de la década de 1930, se desarrolló uno de los primeros y más eficaces mecanismos de reducción de daños. Me refiero a la creación de los grupos de Alcohólicos Anónimos, que no suelen ser incluidos dentro de esta concepción por la mayoría de los que adhieren a esta propuesta, pese a ser un mecanismo comparativamente eficaz, surgido y utilizado por los propios ex consumidores, y más allá de que su objetivo sea la abstinencia.

Subrayo que el levantamiento de la prohibición se aplicó a la principal sustancia considerada adictiva -en ese entonces el alcohol-, que era la "droga" que identificaba y sigue identificando culturalmente a los países occidentales, pero cuya producción y sobre todo su consumo se había expandido notablemente a nivel mundial, incluidos por supuesto América Latina y el Caribe. 
Este antecedente antiprohibicionista es retomado constantemente pero en forma tímida por los que actualmente cuestionan el prohibicionismo, quizá por temor a que en lugar de que este proceso fundamente empíricamente la legalización del resto de las "drogas" genere, por el contrario, un efecto rebote y se vuelva a plantear, si no la prohibición, por lo menos el incremento de actividades punitivas de control respecto de las bebidas alcohólicas, como de hecho lo hemos observado reiteradamente.

\section{POLÍTICAS DE REDUCCIÓN DE DAÑOS: RASGOS Y PROPUESTAS}

Como sabemos, estas propuestas se originaron a través de cuatro vías complementarias. En primer lugar, a través de la experiencia de los propios usuarios de drogas en controlar algunas de las consecuencias más negativas de las mismas, así como en recuperar algunas de las funciones individuales y sociales de su consumo. En segundo lugar, la constatación del fracaso de las políticas prohibicionistas, y la crítica a las consecuencias de todo tipo generadas por dichas políticas, ya que no solo incrementan las consecuencias negativas en términos de salud sino que además vulneran los derechos humanos de los usuarios de esas sustancias prohibidas. Tercero, el reconocimiento de que la legalización no ocurrirá y, por lo tanto, lo "realista" es concentrarse en la reducción de daños. Y cuarto, la necesidad de enfrentar la epidemia de VIH-sida a través de todos los procesos posibles, uno de los cuales es actuar respecto del uso de heroína inyectable (f).

Subrayo que, desde nuestra perspectiva, la reducción de daños ha sido y es realizada por otros grupos de la sociedad civil que no suelen ser incluidos en la reducción de daños, como es el caso de Alcohólicos Anónimos, así como también es generada e impulsada por organismos del Estado, entre ellos el sector salud, y me refiero al establecimiento de medidas como el cinturón de seguridad o el alcoholímetro, que tratan de reducir los daños y los riesgos generados principalmente por el consumo de alcohol. Por lo tanto, incluyo dentro de reducción de daños al conjunto de estas acciones que tienen como objetivo no solo la reducción sino también la prevención de daños (g).

Como ya señalé, algunos países, sobre todo europeos, reconocieron dichas propuestas y se implementaron algunas actividades, en su mayoría impulsadas a través de organizaciones no gubernamentales (ONG). En la mayoría de los países de América Latina, estas políticas no se aplican, se aplican diferencialmente o su aplicación es incipiente y acotada, aun cuando dicha aplicación va en aumento.

Sobre la base de lo mencionado hasta aquí, las principales propuestas y características de las políticas de reducción de daños serían las siguientes $(11,12)$ :

a) Proponer y propiciar cambios en la legislación y en las políticas sobre drogas que incluyan legalizar y despenalizar todas o por lo menos una parte de las drogas. En este aspecto hay diferencias sustantivas entre distintas tendencias de reducción de daños, ya que la casi totalidad propone la legalización de la mariguana $y$, por supuesto, seguir manteniendo la legalización de las bebidas alcohólicas y del tabaco, pero una parte se opone a la legalización de las drogas consideradas más duras como cocaína o heroína.

b) Evitar, reducir y en lo posible eliminar la criminalización de estas drogas y sobre todo de sus consumidores. Reducir hasta eliminar la penalización y persecución de los consumidores.

c) Evitar y reducir hasta eliminar la discriminación y la estigmatización de estas sustancias y de sus usuarios. Eso incluye actuar sobre la autoestigmatización de una parte de los consumidores.

d) Evidenciar, a través de estudios y de la difusión de los mismos en los diferentes niveles de decisión, el fracaso de las políticas y de la mayoría de las actividades prohibicionistas.

e) Difundir los éxitos obtenidos a través de la despenalización de estas sustancias y/o de la aplicación general o específica de actividades de reducción de daños, como son los casos más o menos paradigmáticos de la despenalización Ilevada a cabo por Portugal, o de las consecuencias positivas obtenidas a través de los intercambios de jeringas o de la legalización para uso personal de pequeñas cantidades de sustancias consideradas adictivas. 
f) Cuestionar que los tratamientos médicos y/o las acciones de reducción de daños tengan como único objetivo manifiesto la abstinencia.

g) Sin negar la importancia que puedan tener la atención y prevención biomédicas y psicológicas, cuestionar la medicalización y psiquiatrización del consumo y las consecuencias de estas sustancias, que tienden a excluir el denominado "punto de vista del actor", en este caso del usuario de drogas. Despenalizar el uso de estas sustancias y reconocer que una parte de los usuarios pueden ser tratados biomédicamente -o a través de otras formas de atención-, pero sin convertir ninguna forma de atención en una obligación penalizada.

h) Favorecer que el consumo sea considerado un derecho de los usuarios, un comportamiento "normal" y no patológico, aunque reconociendo que puede tener consecuencias negativas. Por lo cual se propone eliminar la imagen jurídico-policial y favorecer la imagen de consumidor normal, como ocurre en el caso del consumidor de alcohol; o por lo menos favorecer una imagen donde el padecimiento sea entendido como tal y, por lo tanto, eliminar la imagen penal. Desde esta perspectiva se promueven, por una parte, el desarrollo de instituciones médicas y de "asistencia social" específicas con atención profesional (médica, psicológica, etc.) de los sujetos y, por otra, la creación de instituciones como las "Cortes de Drogas" que den un estatus penal diferencial a los usuarios de las sustancias adictivas consideradas ilegales, aunque reconociendo que las mismas pueden tener un uso negativo.

i) A través de diferentes medios educativos, promocionales, preventivos, experienciales, etc., favorecer que el usuario conozca los aspectos positivos y las consecuencias negativas de las diferentes sustancias consideradas adictivas $-y$ sobre todo de las que utiliza- en términos individuales y grupales. Impulsar no solo el conocimiento, sino el manejo por el usuario de los efectos negativos, para reducir sus consecuencias.

j) Observar, a través de los comportamientos de los propios usuarios, las conductas, instrumentos, concepciones que favorezcan la reducción de daños no solo en términos individuales sino grupales. Este es un trabajo que debe ser sistemático buscando todo tipo de posibilidades generadas por los propios usuarios, pues se parte del supuesto de que serían las acciones que serán más aceptadas por otros usuarios pertenecientes, por lo menos, al mismo contexto social.

k) Favorecer la existencia de grupos de usuarios que se organicen y luchen contra la ilegalidad y la penalización, por el derecho al consumo, y por el conocimiento y reconocimiento de las políticas de reducción de daños.

I) Impulsar la construcción y/o reconstitución de redes sociales de protección, de sostén, de ayuda mutua entre los usuarios de las sustancias consideradas adictivas.

m) Reconocer que una parte de los consumidores, aun con graves consecuencias físicas o mentales, no busca ni quiere tratamientos médicos; incluso los rechaza y por lo tanto no va a los servicios de atención de ningún tipo. Lo cual supone pensar y desarrollar otro tipo de estrategias de acción. Si bien las ONG e instituciones de salud han promovido en algunos países la atención y prevención en situación de calle, debemos asumir que una parte de los usuarios, y por muy diferentes razones, rechazan también este tipo de atención. Dichas razones pueden tener que ver con "ocultar" sus actividades de narcomenudeo, porque consideran que los servicios de salud no son eficaces o simplemente porque quieren vivir como viven.

n) La perspectiva de reducción de daños reconoce que el usuario de "drogas" tiene derecho a consumirlas, así como a decidir si busca o no tratamiento o ayuda. Pero este reconocimiento tiene que ser articulado con los daños que el sujeto puede generar no solo a su propio cuerpo sino al cuerpo de los otros. $Y$ este es uno de los puntos más necesarios a analizar y a resolver por lo menos provisionalmente, en términos de la formulación de regulaciones específicas que sobre todo tomen en cuenta las consecuencias del comportamiento del usuario de drogas en los "otros".

o) Los grupos de usuarios de drogas consideradas adictivas y otros sectores de la sociedad civil deberían intervenir -si les interesara- en la planificación, diseño e intervención de las políticas y actividades generadas respecto de los usos y consecuencias de las drogas consideradas adictivas. 
Considero que uno de los roles fundamentales de las propuestas, y sobre todo de las actividades de reducción de daños, ha sido impulsar y contribuir a morigerar la aplicación de las políticas prohibicionistas, incluyendo en forma directa o articulada las concepciones y acciones de los propios usuarios para limitar los daños a la salud, sino también las concepciones y acciones de los propios usuarios. No pueden entenderse las modificaciones generadas en países europeos y americanos al prohibicionismo sin el papel activo de las organizaciones que han impulsado la reducción de daños (h).

Respecto de las propuestas de reducción de daños, se han generado críticas provenientes de diferentes orientaciones. Por una parte, se sostiene que la legalización total o parcial incrementará la producción y el consumo de sustancias adictivas con consecuencias negativas en términos sociales y de salud; lo cual, por ejemplo, tendría repercusiones específicas en el sector salud ya que elevaría los costos de atención y de prevención, así como también incrementaría el desarrollo de otros delitos, especialmente contra las personas, para compensar la reducción de las ganancias generadas por el narcotráfico. Desde otras perspectivas, se señala que el trabajo realizado se centra en individuos o en menor medida en microgrupos $y$, si bien puede considerarse el papel de los procesos económicos y culturales, estos no suelen ser incluidos. Se trata sobre todo de ayudar a los sujetos. En consecuencia, se cuestiona limitar las acciones de reducción de daños a la aplicación de ciertas actividades específicas, en lugar de impulsar una política global de reducción de daños que incluya aspectos estructurales.

\section{PROPUESTAS DE REDUCCIÓN DE RIESGOS}

Las propuestas de reducción de riegos buscan subrayar y profundizar determinados aspectos que, si bien son analizados y accionados a partir de las propuestas de reducción de daños, han sido menos trabajados y utilizados, o directamente no han sido tomados demasiado en cuenta, sobre todo en términos de acción (i).
Estas propuestas asumen las trayectorias de la reducción de daños generadas y aplicadas tanto por las organizaciones de la sociedad civil como por instituciones del Estado y, especialmente, los objetivos pragmáticos de reducir lo más posible determinados daños y aspectos sociales e institucionales que afectan negativamente a los usuarios de las sustancias consideradas adictivas. Y, a partir de dicho pragmatismo, impulsar la articulación entre el conjunto de actores sociales que se organizan en torno a cuestionar las políticas prohibicionistas, en la defensa de los derechos de los consumidores y, en particular, en la aplicación de acciones para reducir daños y riesgos.

Una parte significativa de la reducción de daños refiere a actividades que han sido eficaces como el intercambio de jeringas o la creación de espacios sociales de consumo terapéutico de mariguana. Si bien estas actividades son sumamente importantes por su demostrada eficacia, considero que la reducción de daños no debiera limitarse a acciones de este tipo. Por lo tanto, aunque algunas de las características que enumeraré son reconocidas e impulsadas por algunos sectores que trabajan con reducción de daños, no son utilizadas por otros, e incluso son cuestionadas, y es por ello que las incluyo aquí. Señalo además, que toda una serie de actividades enumeradas en el apartado de reducción de daños, también corresponden a la propuesta de reducción de riesgos, pero articuladas con las que presentaré en este apartado.

Hay toda una serie de experiencias y de investigaciones que avalan la propuesta de reducción de riesgos, y de las cuales solo mencionaré algunas. Me refiero, por ejemplo, a los estudios de Antonovsky $(15,16)$ sobre el papel que cumple el coping (enfrentamiento), pero pensado en términos de grupo y no solo de sujeto; a la existencia de capitales sociales y culturales que posibiliten enfrentar los riesgos, así como a las propuestas de empoderamiento que observamos no solo en el caso del género femenino o de ciertos grupos con $\mathrm{VIH}$-sida, sino a nivel de lo que se conoce como paciente bien informado. Desde estas perspectivas es importante recuperar los trabajos que desde fines de la década de 1940 demostraron el papel que la pertenencia y el activismo tanto político como ideológico tuvieron 
para posibilitar la sobrevivencia de sujetos en situaciones tan límites como fueron los campos de concentración alemanes bajo el nazismo.

Considero que hay una serie importante de trabajos, en parte derivados de las consecuencias de la denominada Segunda Guerra Mundial, que evidencian en distintos aspectos la existencia de recursos individuales y colectivos para reducir daños y riesgos como son los de Bowlby (17), o los que fueron impulsados por la corriente italiana de "la salud en la fábrica" (18). Es decir, la política de reducción de riesgos debería recuperar la corriente de estudios y de experiencias de muy diferente tipo que coloca en las dimensiones sociales, económicas, ideológicas y culturales -y también en los sujetos y microgrupos- las posibilidades de un mayor control de riesgos y de daños. Más aun, considero que debe recuperarse el papel central de la autoatención como uno de los principales procesos que posibilita articular lo individual y lo colectivo en el desarrollo de una política sobre las sustancias consideradas adictivas (19).

A partir de lo señalado, algunas de las características de la reducción de riesgos serían las siguientes:

a) Las drogas consideradas adictivas deben ser analizadas no solo como drogas adictivas en sí, sino como parte de procesos a través de los cuales se construyen, definen y se usan individual y socialmente las diferentes drogas. Si bien asumo que las drogas -y no solo las adictivas- pueden tener consecuencias negativas de muy diferente tipo, reconozco que también pueden cumplir funciones positivas, y que tanto los aspectos negativos como los positivos pueden operar simultáneamente. Durante años he estudiado el "alcoholismo" desde esta perspectiva, y formulado el concepto "proceso de alcoholización", que incluye tanto las funciones positivas como las consecuencias negativas generadas por los usos del alcohol, lo cual implica no reducir el consumo de alcohol a sus aspectos patológicos, pero tampoco negarlos. Y considero que lo mismo podemos hacer respecto de las otras drogas consideradas adictivas $(1,20,21)$.

b) Es en función de esta característica $-y$ de otras- que resulta difícil pensar que exista un "mundo sin drogas", dada la variedad de fun- ciones de muy diferente tipo que cumplen las mismas. Puede ser que ciertas drogas dejen de usarse, que sean incluso eliminadas o tal vez olvidadas, pero al pensar en las drogas adictivas, si bien necesitamos pensar en cada una de ellas, también debemos pensar en las funciones que cumplen en su conjunto y más allá de la especificidad de cada una.

Si algo caracteriza a la sociedad denominada occidental es que posiblemente constituya la sociedad que usa simultáneamente el mayor número y variedad de drogas consideradas adictivas. Más aun, considero que es la sociedad que no solo se ha "apropiado" de sustancias utilizadas por otras sociedades, sino que ha creado gran parte de las drogas adictivas que se consumen legal e ilegalmente en la actualidad. Y las ha creado a través de científicos y técnicos que trabajan en la industria químico-farmacéutica y que son prescriptas médicamente; así como son creadas a través de las reelaboraciones generadas por la sociedad civil como es el caso del "paco", o de las "tachas", pero también de las mezclas tipo "teporocho" o de las que combinan bebidas energizantes o alcohol con otras sustancias adictivas. Los sujetos y grupos, por diversas y frecuentemente contradictorias razones, necesitan consumir este tipo de sustancias, como parte de la sociedad y/o de los grupos sociales a los que pertenecen $y$, por supuesto, por sus propias necesidades y problemas individuales.

c) La reducción de daños incluye, por lo menos en parte, la reducción de riesgos que operan en diferentes procesos de salud-enfermedad. Dicha tendencia ha trabajado sobre la reducción del riesgo de contraer VIH-sida u otros procesos infectocontagiosos, cuando los usuarios usan drogas inyectables. La distribución e intercambio de jeringas constituye una de las actividades que identifica en gran medida la reducción de daños. Como ya lo señalamos, en varios países los propios organismos de seguridad social han impulsado la reducción de daños respecto de drogas supuestamente adictivas pero legales, como es el caso del alcohol, a través del uso obligatorio del cinturón de seguridad o la aplicación del alcoholímetro. Pero la reducción de riesgos y de daños 
no solo debería actuar sobre este tipo de aspectos, sino intervenir $-\mathrm{y}$ de ser posible eliminar- algunos aspectos estructurales de los riesgos como, por ejemplo, el que especialmente los jóvenes de varios países de América Latina se incluyan cada vez más en actividades criminales no solo para conseguir drogas para su propio consumo, sino como parte de su trabajo en el narcotráfico.

Debemos asumir que tanto la inserción en actividades delictivas, como la criminalización de los comportamientos juveniles, así como su participación protagónica en accidentes automovilísticos han contribuido a que, por primera vez, la mortalidad en jóvenes (15 a 24 años) supere a la mortalidad en niños (menos de 10 años) a nivel internacional (22).

Pero además, la mayoría de los jóvenes y de los niños que mueren por violencias o que son detenidos, chantajeados y/o encarcelados son pobres y viven dentro de un círculo de pobreza que se desarrolla y transmite generacionalmente. Si bien la pobreza, la extrema pobreza, o la desocupación crónica no son los únicos factores que impulsan el consumo de drogas adictivas, lo que las caracteriza es que generan un mayor deterioro de los sujetos que las consumen. Por lo cual, las políticas de salud y las políticas sociales deberían tomar como referente casi permanente de sus propuestas y acciones, además de la pobreza, al "círculo de pobreza" del que forma parte el consumo de ciertas drogas legales e ilegales. Debemos tratar de romper el "círculo de la pobreza" para crear las condiciones de reducción de daños y de riesgos que vayan más allá de las condiciones de cada sujeto $(23,24)$. Debería incluirse la posibilidad de establecer -como lo han propuesto diversos autores desde la década de 1930 hasta la actualidad- un nivel mínimo de vida que posibilite realmente no solo ingresos sino vivienda, educación, seguridad, esparcimiento. De tal manera que, a partir de un determinado nivel de vida, deberíamos observar las consecuencias de los usos de drogas adictivas y no adictivas, recordando que la legalización de las drogas no modifica el hecho de que, sobre todo, ciertos grupos pobres continúen siendo los más vulnerables, como ha sido evidenciado históricamente a través del consumo de alcohol.
Por lo cual, la reducción de riesgos implica incluir las condiciones económicas, sociales y culturales como parte sustantiva de una política real de reducción de daños ya que, si no se modifican radicalmente ciertos aspectos de la realidad social, al menos determinados riesgos y daños se incrementarán. Solo la legalización posibilitará una reducción de daños y de riesgos efectivas: "A menos que revoquemos la prohibición de las drogas y todo el bagaje de actitudes públicas que Ileva consigo, las cosas continuarán empeorando" (25 p.118) (j).

d) Debemos trabajar con los procesos estructurales, incluyendo especialmente la Ilamada violencia estructural -que otros Ilaman sistémica- en el análisis, en las propuestas y en la intervención, lo cual implica tomar centralmente en cuenta la causalidad tanto a nivel individual como social. Si bien una parte de la reducción de daños tiene en cuenta la causalidad, la misma pasa a ser secundaria dado que sus objetivos son básicamente pragmáticos, es decir, reducir daños puntuales; mientras que, para la reducción de riesgos, constituye uno de los núcleos teórico y empírico fuertes.

e) Trabajar, por lo tanto, no solo con la vulnerabilidad en términos físicos y psíquicos, sino trabajar con la vulnerabilidad social y económica de los sujetos, incluyendo las vulnerabilidades específicas desarrolladas por su pertenencia de clase social, de género, de edad, de grupos étnicos. Lo cual, y lo aclaro, no supone reducir nuestras acciones a los determinantes sociales, sino incluir también los factores de riesgo en términos inclusive de estilo de vida, pero referidos a conjuntos sociales y a conductas intencionales y no intencionales.

f) Incluir una perspectiva de salud pública, de medicina social, de derechos humanos, pero también de antropología médica, que repiense las políticas y actividades respecto de los usos de las sustancias ilegales consideradas adictivas, que vaya más allá de las mismas. Es decir que, en términos de sustancias, equipare la situación de estas sustancias no solo con el alcohol y el tabaco, sino con la situación de los usos sociales y de las acciones estatales respecto de la sal, del azúcar, de las farináceas, de las carnes rojas, de los alimentos 
"basura" o "chatarra" que han pasado a ser parte de las principales causas de mortalidad, dado su papel decisivo en la mortalidad por diabetes mellitus o por enfermedades cardiovasculares. En términos de salud pública y dadas sus consecuencias crecientes en la mortalidad, la sal, los azúcares o los alimentos "basura" deberían ser tan o más prohibidos que las drogas consideradas adictivas. Pero, pese a los daños que generan y a los riesgos que implican, dichas sustancias no han sido prohibidas sino que se impulsan programas y actividades que asumen el libre consumo de los sujetos, aunque generando recomendaciones y estableciendo algunas condiciones que posibiliten limitar los daños; lo que también debería operar respecto de cada una de las sustancias consideradas adictivas (26).

g) Si determinados procesos existen y se difunden no es solo por los deseos y necesidades de los sujetos, ni porque nos los imponen los medios de comunicación masiva, sino porque operan procesos económico-políticos y/o simbólicos que favorecen su surgimiento y desarrollo. La gran expansión del consumo de drogas no podría entenderse sin incluir ciertas consecuencias contradictorias del neoliberalismo desarroIladas durante las décadas de 1980 y 1990. El narcotráfico, basado en la ilegalidad de ciertas sustancias, no podría desarrollarse y expandirse si no contara con una base social de apoyo, con un "ejército de reserva" para realizar las diferentes actividades "delictivas" y no delictivas, así como con el establecimiento de relaciones directas e indirectas tanto con las esferas de gobierno como con sectores de la sociedad civil, favorecidos, por supuesto, por las condiciones generadas por el neoliberalismo. No debemos olvidar que la mayoría de las ganancias obtenidas en forma ilegal por el crimen organizado se "lavan" a través del aparato productivo, comercial y financiero legal.

h) Promover y acompañar la lucha de los grupos organizados en torno a la defensa de los derechos de los consumidores de drogas consideradas adictivas y, en lo posible, promover la articulación de las luchas desarrolladas por las mujeres, por las personas con VIH-sida, por los grupos gay, por los grupos étnicos y por otros grupos que se organicen a partir de su especificidad y diferencia. Si bien reconozco que las características de estos grupos implican luchas diferentes por objetivos diferentes, y que sus luchas cobran eficacia sobre todo a partir de luchar por su especificidad, debemos observar las coyunturas y los procesos que posibiliten esas convergencias, como ocurre con la defensa de la coca por el actual gobierno boliviano, o la convergencia que se dio en los hechos entre sectores gay y consumidores de drogas inyectables, por lo menos en ciertos contextos.

i) Esta propuesta, si bien incluye centralmente el punto de vista del actor, como lo hemos señalado reiteradamente (14), no reduce la comprensión e intervenciones solo al punto de vista del actor. Necesitamos incluir tanto los testimonios, experiencias y prácticas del consumidor, como los de otros actores entre los que destaco tres: los que cuestionan a los consumidores, los que son dañados por los consumidores y los que acompañan a los consumidores tratando de mejorar su situación y que no solo se reducen a las ONG sino que incluyen sectores gubernamentales tanto de tipo biomédico como de otros campos.

j) Estas propuestas deben cuestionar y evidenciar las consecuencias de las políticas prohibicionistas en la calidad de vida de los consumidores, en la promoción de la estigmatización, discriminación y criminalización, así como en el desarrollo de procesos de corrupción y de impunidad.

k) Un aspecto central es el reconocimiento de que las políticas prohibicionistas se manejan en varios niveles respecto de la producción, comercialización y uso de las diferentes drogas consideradas adictivas legales e ilegales. Un primer nivel refiere a políticas diferentes para drogas consideradas adictivas, como ya vimos, respecto de las bebidas alcohólicas y tabaco comparados con el resto de las drogas consideradas adictivas. Un segundo nivel refiere a analizar conjuntamente las consecuencias en la salud de la población generadas por el consumo de sustancias adictivas y no adictivas, como también lo señalamos previamente. $Y$ un tercer nivel refiere a analizar comparativamente la producción, comercialización, uso y consecuencias de las drogas consideradas adictivas ilegales y las "legales" 
producidas por la industria químico-farmacéutica y que deben ser obligatoriamente adquiridas con recetas médicas.

l) En función de lo señalado, una de las estrategias centrales de la reducción de riesgos debería colocar en primer plano los aspectos científicos, profesionales y técnicos para discutir los diferentes aspectos que legitiman las acciones de los Estados para ilegalizar, prohibir, encarcelar, discriminar, así como para legalizar las diferentes sustancias consideradas adictivas. Debe analizarse en términos científicos la real calidad adictiva de estas drogas, así como qué significa la dependencia a las mismas. Pero también deben compararse epidemiológicamente las consecuencias en la morbilidad y mortalidad con otros aspectos sociales generados por las drogas adictivas y las no adictivas. Debe analizase la información existente para evidenciar en términos científicos las incongruencias y contradicciones de las actuales políticas prohibicionistas (k). Los informes más recientes de los organismos encargados de supervisar la producción y consumo de drogas adictivas en EE.UU. concluyen que en la última década se habría reducido el consumo de la mayoría de las drogas ilegalizadas, pero que se habrían incrementado notablemente la producción y el consumo de las "drogas recetadas" por los médicos, que han pasado a ser las sustancias adictivas más consumidas luego de la mariguana, considerando que "el abuso de drogas recetadas es el problema de drogas que más rápido crece en el país" (28). Pero esta prescripción médica no solo se incrementa en EE.UU., sino también en los países europeos para cuadros depresivos, de estrés, para "sentirse bien" o para acrecentar rendimientos. Este incremento, y es lo que me interesa subrayar, ha generado un aumento de las muertes por sobredosis por dichas drogas. Según un informe del Centro para el Control y la Prevención de Enfermedades de EE.UU., la muerte por sobredosis de drogas recetadas es mayor que la generada por el consumo de drogas ilegales ya que, mientras las primeras generan 15.000 muertes por año, el resto de las drogas generan 12.000 muertes, de las cuales 4.000 son por sobredosis de heroína y cocaína $(29,30)$.
Debe colocarse en primer plano $-\mathrm{y}$ no ocultarlo o negarlo- como parte de una política de reducción de riesgos que, por ejemplo, el alcohol constituye la sustancia considerada adictiva, aunque legalizada, que más consecuencias negativas tiene en términos de morbilidad y mortalidad a través de la cirrosis hepática, el síndrome de dependencia alcohólica (I) y el síndrome de alcoholismo fetal por una parte; $y$, por otra, es la sustancia más relacionada con violencias tanto en términos de homicidios como de muertes por accidentes automovilísticos. Esto no solo es sabido por los especialistas en "alcoholismo" y por el saber médico en general $(1,2)$ desde hace mucho tiempo, sino que reiteradamente se documenta a través de la información epidemiológica y/o de investigaciones específicas.

Recientemente Nutt, King y Phillips (31), publicaron los resultados de una investigación en la cual compararon los efectos negativos de 20 drogas consideradas adictivas, concluyendo que el alcohol es por mucho la más dañina de dichas drogas a nivel internacional $(m)$. Lo señalado debe conducir a cuestionar las representaciones sociales, los discursos y especialmente las prácticas dominantes acerca de que las drogas adictivas ilegalizadas constituyen un grave problema, para demostrar que es sobre todo un problema construido.

m) No reducir los objetivos de la reducción de riesgos a la abstinencia, pero no cuestionar la abstinencia si los sujetos y grupos lo deciden intencionalmente respecto de su propio consumo.

n) Observar los logros y la eficacia de las políticas prohibicionistas y las de reducción de daños así como las consecuencias negativas y positivas de las mismas, teniendo como referente las condiciones de los consumidores y de la sociedad en general. Aclarar por lo tanto, que si bien la legalización elimina o reduce daños y riesgos, no solo no reduce otros, sino que en algunos casos los incrementa.

o) Desde la concepción que presento, las políticas prohibicionistas constituyen una suerte de incongruencia en las sociedades capitalistas caracterizadas, sobre todo en la etapa neoliberal, por impulsar el consumo, dado que las políticas prohibicionistas lo que tratan es de eliminar o por lo menos reducir el consumo 
de algunas de las sustancias consideradas adictivas. De tal manera que esta política no solo cuestiona y se opone a las leyes del mercado, sino que no podría explicar por qué durante la etapa neoliberal es cuando más se expande la producción y consumo de sustancias adictivas de todo tipo.

Hay un último aspecto que me interesa recuperar y que ya señalé en el texto. Me refiero al de analizar si realmente las políticas prohibicionistas y, en particular, la "guerra a las drogas" han fracasado. Si bien desde la década de 1990 se viene constatando y señalando dicho fracaso, la cuestión está en observar si los objetivos "reales" de esta guerra fueron y/o son la eliminación -o por lo menos reducción- del consumo de drogas, o si sus objetivos tienen que ver con el desarrollo de justificaciones de control e intervención permanente y coyuntural, especialmente sobre ciertos grupos y sobre ciertos países, cuando se considere necesario.

Si bien, desde 1914, EE.UU. emprendió una lucha contra las drogas consideradas adictivas con más fuerza que ningún otro país, dicha lucha se caracterizó por su bajo perfil, expresado en gran medida por los limitados recursos aplicados a la misma. Pero a partir de la presidencia de Richard Nixon, y por diversos factores, se reimpulsó esta lucha convirtiéndose en "guerra a las drogas":
El gobierno de los EE.UU. nunca antes había puesto en práctica una medida de presión política y económica de esa naturaleza a causa de las drogas [...] Nixon había encontrado en una estrategia de perfil relativamente bajo durante décadas, una razón de peso para una intervención más directa y decidida en asuntos internos de otras naciones. (32 p.359)

En síntesis, cuestionamos las políticas prohibicionistas aplicadas a las drogas consideradas adictivas, y proponemos impulsar la reducción de daños pero, sobre todo, la reducción de riesgos a través de una posible articulación entre procesos que operen tanto a nivel macro como microsocial. Un trabajo básico sería detectar quiénes son los actores sociales que se oponen y quiénes los que impulsan estas políticas y actividades, dado que considero que las drogas son básicamente lo que los conjuntos sociales y los sujetos en sus relaciones sociales $y$, especialmente en sus relaciones de hegemonía/subalternidad, hacen con las drogas, y no solo lo que las drogas hacen con los sujetos y grupos. Y justamente EE.UU. y, por supuesto, otros países, han usado las drogas consideradas adictivas en función de determinados objetivos, por lo que han convertido las drogas en un mecanismo de control político y social.

\section{NOTAS FINALES}

a. América Latina, y especialmente ciertos países y regiones, se ha caracterizado históricamente por sus altas tasas de homicidio debidas a diversos procesos y factores económicos, políticos y culturales. $\mathrm{Y}$ es sobre esta tendencia que el crimen organizado impulsa sus violencias homicidas.

b. Este texto, si bien hace referencias constantes a otros contextos, está pensado para las situaciones latinoamericanas y especialmente para México. Mis análisis y propuestas están basados sobre todo en mis trabajos sobre el proceso de alcoholización en México $(1,2)$. c. Necesitamos asumir que el consumo de ciertas sustancias consideradas adictivas es "legalizado" cuando el mismo se convierte en consumo terapéutico, como por ejemplo en los casos de la mariguana y de la metadona; $y$, sobre todo, a través de la interminable lista de sustancias consideradas adictivas, pero que son recetadas por los médicos.

d. Deben subrayarse dos hechos: primero, que la conversión de las drogas en problema de salud en lugar de problema delictivo, si bien medicaliza, posibilita modificar algunos de los aspectos más negativos del prohibicionismo. $Y$, segundo, la necesidad de distinguir entre el sector salud $y$ la investigación biomédica y sociomédica, dado que mientras el primero adhiere a alguna de las 
variadas formas de prohibicionismo, una parte de la investigación médica y social analiza y denuncia las incongruencias y falacias de las políticas prohibicionistas. Considero que cada vez más, las políticas prohibicionistas tratarán de justificarse a través de criterios biomédicos.

e. Tanto los conceptos de adicción como de dependencia no resisten la crítica teórica ni empírica $(1,6)$.

f. Mientras las tres primeras vías son propuestas e impulsadas por usuarios, ONG e intelectuales, la cuarta es la que realmente condujo a los gobiernos a apoyar la reducción de daños, y especialmente ciertas actividades como el intercambio de jeringas.

g. Si bien es importante reconocer los orígenes de la denominada reducción de daños, en términos de las acciones y concepciones que sobre todo respecto de los usuarios de drogas tienen los sectores de la sociedad civil que la crearon e impulsaron, considero sin embargo necesario incluir las acciones de reducción de daños impulsadas por otros sectores más allá de su origen social o estatal. Lo cual no implica que dichas acciones y organizaciones se unifiquen, sino que funcionan articuladamente a partir de las motivaciones y formas de organización particulares. No obstante, deberíamos asumir en todo lo que significa, que el financiamiento de actividades por parte de los gobiernos ha ido articulando en los hechos a los sectores estatal y social, salvo en el caso de algunos grupos de autoayuda.

h. Una gran parte de las actividades de reducción de daños son realizadas en los países europeos por ONG o similares, que logran su financiamiento a través de organismos internacionales y/o de los gobiernos de cada país. En países como España, donde operan unas 250 organizaciones sociales de atención a drogodependientes cuyo financiamiento se basa en los gobiernos autonómicos, dada la actual crisis económica las ONG podrían quedar sin esos aportes financieros. Como señala el actual director de la Fundación de Ayuda contra la Drogadicción: "Las ONG dependen de fondos públicos; si no llegan y desaparece esa red de cobertura, el problema puede descontrolarse y los drogodependientes pueden volver a la calle" (13). Y éste es un problema que requiere ser pensado a fondo.

i. El concepto reducción de riesgos ha sido cuestionado, incluso por mí, en la medida en que las propuestas dominantes piensan tanto el riesgo como la reducción del mismo en términos de responsabilidad individual e intencional, dejando de lado las condiciones estructurales que están operando no solo a nivel del contexto sino del mismo sujeto. Pero el hecho de que haya sido utilizado así, sobre todo por el sector salud, no impide que nosotros lo usemos a partir de una perspectiva que incluya centralmente los aspectos estructurales y los actores sociales, sin eliminar-obviamente- sus responsabilidades en las consecuencias generadas (14).

j. Es obvio que no solo los pobres consumen, ni son los principales consumidores de sustancias consideradas adictivas; así como tampoco son los que tienen siempre una participación mayor en los accidentes viales. Pero sí son los que tienen las consecuencias más negativas y frecuentemente irreversibles.

k. Por supuesto que asumimos que el conocimiento en sí y por más que evidencie con criterios y datos "objetivos" la verdad, no es sin embargo quien decide la verdad respecto al tipo de proceso que estamos tratando, sino que la decide -con palabras a la moda- el "poder", como tempranamente lo planteó Dilthey, quien sostuvo que es la "facticidad" la que resuelve en la práctica la verdad o falsedad de un hecho $(14,27)$. Lo cual no implica que no sigamos señalando, contrastando y demostrando cuáles son los criterios "objetivos" en términos de conocimiento y de ser posible de intervención.

I. Las cinco encuestas nacionales realizadas en México desde 1986 sobre consumo de drogas consideradas adictivas concluyen que el alcohol es la sustancia que "produce" más sujetos "dependientes" comparada con cualquier otra droga. Más aun, durante varios años en las décadas de 1980 y 1990, el síndrome de dependencia alcohólica estuvo entre las primeras veinte causas de muerte en varones, lo cual no ocurre con ninguna otra droga.

m. Las 20 sustancias fueron valoradas del 0 al 100 -siendo 100 el nivel máximo de dañogenerando los siguientes resultados: alcohol 72, heroína 55, crack 54, metanfetamina en cristal 33 , cocaína 27, tabaco 26, anfetaminas 23, cannabis 20, ácido gama-hidroxibutírico 18 , benzodiacepinas 15, quetamina 15, metadona 14, mefedrona 13 , butano 10 , khat 9, éxtasis 9, esteroides anabolizantes $9, \operatorname{LSD} 7$, bupremorfina 6, y setas 5. Para esta evaluación se aplicaron 16 criterios. Debe subrayarse que reiteradamente se señalan datos que científicamente establecen que determinadas drogas son consideradas nocivas, pese a ser mucho menos negativas para la salud que otras que han sido ilegalizadas (4). 


\section{REFERENCIAS BIBLIOGRÁFICAS}

1. Menéndez EL. Morir de alcohol. saber y hegemonía médica. México DF: Alianza Editorial Mexicana; 1990.

2. Menéndez EL, Di Pardo RB. De algunos alcoholismos y algunos saberes. Atención primaria y proceso de alcoholización. México DF: Ciesas; 1996.

3. Becker H. Los extraños: Sociología de la desviación. Buenos Aires: Tiempo Contemporáneo; 1971.

4. Grisnpoon L. Reconsideración de la marihuana. México DF: Extemporáneos; 1973.

5. Jelsma M. Panorama internacional de reformas políticas de drogas. En: Touzé G, Goltzman P, compiladores. América Latina debate sobre drogas. Buenos Aires: Intercambios; 2011. p. 331-344.

6. Edwards G, Arif A, coordinadores. Los problemas de la droga en el contexto sociocultural: una base para la formulación de políticas y la planificación de programas. Ginebra: OMS; 1981. (Cuadernos de Salud Púbica, 73).

7. Nadelman E. Buenas y malas noticias en el debate sobre drogas. En: Touzé G, Goltzman P, compiladores. América Latina debate sobre drogas. Buenos Aires: Intercambios; 2011. p. 361-366.

8. Boiteux L, Corda A, Edward SG, Garibotto G, Giacoman D, Guzmán D, et al. Sistemas sobrecargados: Leyes de drogas y cárceles en América Latina. Washington: TNI, WOLA; 2010.

9. Organización de las Naciones Unidas. Resumen [Internet]. World Drug Report 2008 [citado 12 dic 2011]. Disponible en: http://www.unodc.org/documents/wdr/WDR 20 08/wdr08_execsum_spanish.pdf

10. Tokatian JG. Diagnóstico global y oportunidades para gestar un nuevo paradigma en la cuestión de las drogas. En: Touzé G, Goltzman P, compiladores. América Latina debate sobre drogas. Buenos Aires: Intercambios; 2011. p. 31-38.

11. O'Hare PA, Newcombe $R$, Matthews A, Buning EC, Drucker E. La reducción de los daños relacionados con las drogas. Barcelona: Grup IGIA; 1995

12. Romaní O. Las drogas: Sueños y razones. Barcelona: Ariel; 1999.
13. González A. Los impagos ponen en riesgo la ayuda a los drogodependientes. Público [Internet]. 14 nov 2011 [citado 2 dic 2012]. Disponible en: http://www.publico.es/espana/406743/los-impagos-ponen-en-riesgo-laayuda-a-los-drogodependientes

14. Menéndez EL. La parte negada de la cultura: Relativismo, diferencias y racismo. 2da ed. Rosario: Prohistoria ediciones; 2010.

15. Antonovsky A. Social class, life expectancy and overall mortality. Milbank Memorial Found Quart. 1967;45:37-48.

16. Antonovsky A. Health, stress and coping: new perspectives on mental and physical wellbeing. San Francisco: Jessey-Bass; 1979.

17. Bowlby M. Maternal care and mental health. Geneva: World Health Organization; 1951.

18. Basaglia R, et al. La salute in fabbrica: Per una línea alternativa di gestione della salute nei posti di lavoro e nei quartieri. Roma: Savelli; 1974.

19. Menéndez EL. Hacia una práctica médica alternativa. Hegemonía y autoatención (gestión) en salud. México DF: CIESAS; 1982. (Cuadernos de la Casa Chata, 86).

20. Cortés B. La funcionalidad contradictoria del consumo colectivo de alcohol. Nueva Antropología. 1988;34:157-186.

21. Menéndez EL. El proceso de alcoholización: Revisión crítica de la producción socioantropológica, histórica y biomédica en América Latina. Revista Centroamericana de Ciencias de la Salud. 1982;22:61-94.

22. Viner RM, Coffey C, Mathers C, Bloem P, Costello A, Santelli J, Patton GC. 50-year mortality trends in children and young people: a study of 50 low-income, middle-income and high-income countries. The Lancet. 2011;377(9772):1162-1174.

23. Massé R. Culture et santé publique. Montreal: Gaëtan Morin Editeur; 1995.

24. Toussignant M. La pauvreté: cause ou espace des problems de santé mentale. Santé Mentale au Quebec. 1989;XIV(2):91-104.

25. Drucker E. Las políticas de drogas de los Estados Unidos: Salud Pública versus prohibición. En: O'Hare PA, Newcombe R, Matthews A, Buning EC, Drucker E. La reducción de los daños relacionados con las drogas. Barcelona: Grup IGIA; 1995. p. 107-120. 
26. Menéndez EL, Di Pardo RB. Alcoholismo, otras adicciones y varias imposibilidades. En: Minayo MCS, Coimbra Jr. CE, organizadores. Críticas e atuantes: ciências sociais e humanas em saúde na América Latina. Rio de Janeiro: Fiocruz; 2005. p. 567-586.

27. Barth H. Verdad e ideología. México DF: Fondo de Cultura Económica; 1951.

28. Alertan de abuso de drogas legales. Reforma. 11 jul 2011:Sec. Internacional.

29. BBC Salud. Los analgésicos causan más muertes en EE.UU. que la cocaína. BBC Mundo [Internet]. 02 nov 2011 [citado 12 dic 2011].
Disponible en: http://www.bbc.co.uk/mundo/noticias/2011/11/111102_epidemia_analgesicos_eeuu _men.shtml

30. Brooks D. En EU, las muertes por fármacos recetados superan los decesos por heroína y cocaína. La Jornada [Internet]. 21 abr 2011 [citado 12 dic 2011]. Disponible en: http://www.jornada.unam.mx/2011/04/21/mundo/019n1mun

31. Nutt D, King L, Phillips L. Drug harms in the UK: a multicriteria decision analysis. The Lancet. 2010;376(9752):1558-1565.

32. Astorga L. Drogas sin fronteras. México DF: Grijalbo; 2003.

\section{FORMA DE CITAR}

Menéndez EL. Sustancias consideradas adictivas: prohibición, reducción de daños y reducción de riesgos. Salud Colectiva. 2012;8(1):9-24.

Recibido el 4 de febrero de 2012

Aprobado el 19 de marzo de 2012 